\title{
Revisiting Samuelson's models, linear and nonlinear, stability conditions and oscillating dynamics
}

\author{
Fabio Tramontana $^{1 *}$ (D) and Laura Gardini ${ }^{2}$
}

\author{
${ }^{*}$ Correspondence: \\ fabio.tramontana@unicatt.it \\ ${ }^{1}$ Department DiMSEFA \\ Università Cattolica del \\ Sacro Cuore, Via Necchi, n.9, \\ 20123 Milano, Italy \\ Full list of author information \\ is available at the end of the \\ article
}

\begin{abstract}
In this work, we reconsider the dynamics of a few versions of the classical Samuelson's multiplier-accelerator model for national economy. First we recall that the classical one with constant governmental expenditure, represented by a linear second-order difference equation, is able to generate oscillations converging to the equilibrium for a wide range of values of the parameters, and give its analytic solution for all the possible cases. A delayed version proposed in the recent literature, represented by a linear third-order difference equation, is also considered. We show that also this model is able to produce converging oscillations, and give a complete analysis of the stability region of the equilibrium. A new simple nonlinear model is proposed, showing that it keeps oscillatory behavior, although coupled with other dynamics related to global effects. Our analysis confirms that the seminal work of Samuelson and simple modifications of it, may give powerful tools in the study of the business cycles.
\end{abstract}

Keywords: Economic modelling, Samuelson model, Difference equations, Stability of the equilibrium, Oscillatory dynamics, Linear and nonlinear models

\section{Introduction}

There are many economic theories associated with the business cycle (for example, the real business cycle theory (RBC), the dynamic stochastic general equilibrium model (DSGE), Goodwin's growth-cycle model, the Harrodian model, the Kaleckian model, and others), but one of the most fruitful theories, investigated by many authors since its first publications, is certainly the Samuelson's business cycles. In the last decades the seminal work of Samuelson (1939) inspired many scholars in the study of the dynamics related to this model, as well as to its various modifications.

There are recent contributions related to smooth system dealing with many countries or introducing some delay (see e.g. Dassios et al. (2014), Dassios and Zimbidis (2014), Dalla and Varelas (2016), Dassios and Devine (2016), Kostarakos and Kotsios (2017)), as well as papers related to smooth or piecewise smooth variants. In fact, although very simple and proposed many years ago, the Samuelson model still provides answers and rationales to the problem of the business cycles. On the same line, many scholars proposed similar systems, both in continuous time and in discrete time, as well as models

(c) The Author(s) 2021. This article is licensed under a Creative Commons Attribution 4.0 International License, which permits use, sharing adaptation, distribution and reproduction in any medium or format, as long as you give appropriate credit to the original author(s) and the source, provide a link to the Creative Commons licence, and indicate if changes were made. The images or other third party material in this article are included in the article's Creative Commons licence, unless indicated otherwise in a credit line to the material. If material is not included in the article's Creative Commons licence and your intended use is not permitted by statutory regulation or exceeds the permitted use, you will need to obtain permission directly from the copyright holder. To view a copy of this licence, visit http://creativeco mmons.org/licenses/by/4.0/. 
related to smooth or piecewise smooth variants. For example, we can appreciate Puu et al. (2005), Westerhoff (2006), Hommes (1991), Puu (1989, 1997, 2003), Bischi et al. (2019), Rosser (2000). Among the recent works see e.g. Westerhoff (2006), Dassios and Baleanu (2018), Dassios (2018), Barros and Ortega (2019), Ortega and Barros (2020). In particular, the last two papers mentioned above inspired our work. Our goal here is to reconsider the original model and some simple modifications of it, showing that these may represent the dynamics of business cycles.

We first recall that the original Samuelson's model, with constant governmental expenditure, which is described by a linear second-order difference equation, is capable to produce oscillations converging to the equilibrium value of the national income for different values of the parameters. We also consider the delayed version of Samuelson's model presented in Barros and Ortega (2019), which is described by a linear third-order difference equation. It also reveals its capability to produce oscillations converging to the equilibrium value, but for a smaller set of values for the parameters. Moreover, in order to describe a more realistic situation, we propose a simple nonlinear reformulation of the original Samuelson's model, with non constant governmental expenditure, and maintaining the delayed version presented in Barros and Ortega (2019). Our assumption on the governmental expenditure is that at each period it depends on some fixed costs plus a bounded quantity proportional to the income of the previous period. This simple nonlinear version of the Samuelson's model also evidences its ability to represent oscillations converging to the equilibrium value, although these may coexist with other global phenomena.

After this introduction, the rest of the paper is organized as follows. In Sect. 2 we reconsider the classical Samuelson's model and recall how its stability region is obtained depending on the two parameters, namely multiplier and accelerator, and give also its explicit analytic solution. In Sect. 3 we consider the delayed version of Samuelson's model presented in Barros and Ortega (2019). In that work the authors only determine the existence of the equilibrium point. Here we give the analytical description of its stability region, showing that it is in fact mainly associated with oscillatory dynamic behavior. In Sect. 3 we propose our simple nonlinear reformulation of the delayed version of Samuelson's model obtained introducing non constant governmental expenditure. The resulting nonlinear third-order difference equation has an attracting equilibrium for a wide region of values of the parameters, numerically detected. Some simulations show that the dynamics may converge to the equilibrium via oscillatory behavior, being accompanied by coexisting global phenomena which are related to the basin of attraction of the equilibrium. Section 4 concludes.

\section{Method}

\subsection{Original Samuelson's model}

The original model by Samuelson is based on the following assumptions.

Assumption (A). National income $T_{k}$ at time $k$ is given by the sum of three elements: consumption $C_{k}$, private investment $I_{k}$ and governmental expenditure $G_{k}$ :

$$
T_{k}=C_{k}+I_{k}+G_{k}
$$


Assumption (B). Consumption $C_{k}$ at time $k$ depends on the past income and on marginal tendency to consume, modelled by a parameter $c_{1}$, which is the multiplier coefficient, where $0<c_{1}<1$ :

$$
C_{k}=c_{1} T_{k-1}
$$

Assumption (C). Private investment $I_{k}$ at time $k$ depends on consumption changes and on the accelerator factor $b$, where $b>0$. Consequently, $I_{k}$ depends on national income changes:

$$
I_{k}=b\left(C_{k}-C_{k-1}\right)=c_{1} b\left(T_{k-1}-T_{k-2}\right)
$$

Assumption $(D)$. Governmental expenditure $G_{k}$ at time $k$ remains constant, $\bar{G}$.

Hence, the national income is determined by the following second-order linear difference equation:

$$
T_{k+2}-c_{1}(1+b) T_{k+1}+c_{1} b T_{k}=\bar{G}
$$

which, by defining $x_{k}=T_{k}, y_{k}=T_{k+1}=x_{k+1}$, leads to the $2 \mathrm{D}$ system

$$
\left\{\begin{array}{c}
x_{k+1}=y_{k} \\
y_{k+1}=-b c_{1} x_{k}+c_{1}(1+b) y_{k}+\bar{G}
\end{array}\right.
$$

whose fixed point is given by $x=y=\frac{\bar{G}}{1-c_{1}}$, that is:

$$
T^{*}=\frac{\bar{G}}{1-c_{1}}
$$

The stability is determined by the roots of the characteristic polynomial:

$$
\mathcal{P}(\lambda)=\lambda^{2}-c_{1}(1+b) \lambda+b c_{1}=0
$$

which are given explicitly as follows:

$$
s_{1}=\frac{c_{1}(1+b)+\sqrt{c_{1}^{2}(1+b)^{2}-4 c_{1} b}}{2}, s_{2}=\frac{c_{1}(1+b)-\sqrt{c_{1}^{2}(1+b)^{2}-4 c_{1} b}}{2}
$$

The stability occurs iff both eigenvalues are inside the unit circle. The stability region as a function of the two parameters is determined by the conditions $\mathcal{P}(1)=1-c_{1}>0$, which is always satisfied, $\mathcal{P}(-1)=1+c_{1}(1+2 b)>0$, which is satisfied for $b>-\frac{1}{2}-\frac{1}{2 c_{1}}$ and $\mathcal{P}(0)<1$, which is satisfied for $b c_{1}<1$. Thus, for any given $0<c_{1}<1$ and $b>0$, the system is stable for $0<b<\frac{1}{c_{1}}$. Inside the stability region the eigenvalues may be real or complex. From the discriminant $\Delta=c_{1}^{2}(1+b)^{2}-4 c_{1} b=c_{1}\left(c_{1}(1+b)^{2}-4 b\right)$ we have that the roots are complex conjugate when $\Delta<0$, that is, for any fixed value of $c_{1} \in(0,1)$ the roots are complex conjugate for $b_{-}\left(c_{1}\right)<b<b_{+}\left(c_{1}\right)$ where

$$
b_{-}\left(c_{1}\right)=\frac{2-c_{1}-2 \sqrt{1-c_{1}}}{c_{1}}<1, \quad b_{+}\left(c_{1}\right)=\frac{2-c_{1}+2 \sqrt{1-c_{1}}}{c_{1}}>\frac{1}{c_{1}}>1
$$

Notice that the two branches meet at $c_{1}=1$ giving $b_{-}(1)=b_{+}(1)=1$, so that for any fixed value of $c_{1} \in(0,1)$ at the bifurcation related to $b=\frac{1}{c_{1}}$ the fixed point is a center 


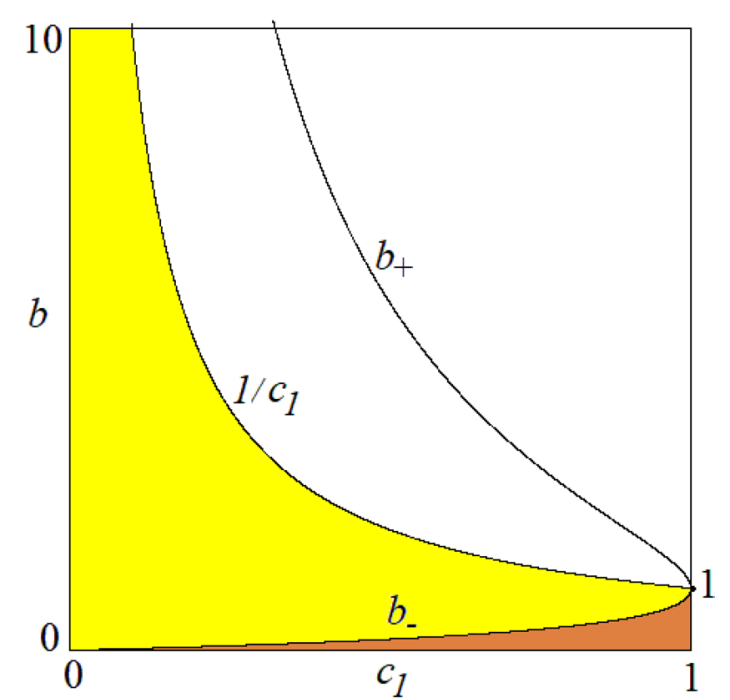

Fig. 1 In color the stability region of the fixed point $T^{*}=\frac{\bar{G}}{1-c_{1}}$ is shown. In the yellow (resp. orange) region the eigenvalues are complex conjugate (resp. real)

and for $b>\frac{1}{c_{1}}$ it is a repelling focus. In Fig. 1 we show in color the stability region in the plane $\left(c_{1}, b\right)$, in yellow the region in which the fixed point is a stable focus, in orange the region in which it is a stable node.

Since the system in (4) is linear, we can write in explicit form its solution, in the considered range $c_{1} \in(0,1)$ and $b<\frac{1}{c_{1}}$ in which the fixed point is stable. As we know from elementary calculus of difference equations (see. e.g. Gumowsky and Mira (1980), Elaydi (2005), only to cite a few) the second-order difference equation in (4) depends on two arbitrary constants which are determined by using the initial conditions, say $T_{0}$ and $T_{1}$ for $k=0$ and $k=1$, respectively. Then, the solution is given for any $k$, i.e. $T_{k}$ for $k \geq 2$, by the general solution of the homogeneous equation plus a particular solution, which in our case is simply the constant solution, i.e. the fixed point $T^{*}=\frac{\bar{G}}{1-c_{1}}$. The solution of the homogeneous equation depends on the kind of eigenvalues, real or complex conjugate. When the eigenvalues $s_{1}$ and $s_{2}$ are real and distinct (i.e. for $0<c_{1}<1$ and $0<b_{-}\left(c_{1}\right)<1$, orange region in Fig. 1), then the solution, for $k \geq 2$, is given as follows:

$$
T_{k}=\beta_{1} s_{1}^{k}+\beta_{2} s_{2}^{k}+T^{*}
$$

where

$$
\beta_{1}=\frac{-s_{2}\left(T_{0}-T^{*}\right)+\left(T_{1}-T^{*}\right)}{s_{1}-s_{2}}, \beta_{2}=\frac{s_{1}\left(T_{0}-T^{*}\right)-\left(T_{1}-T^{*}\right)}{s_{1}-s_{2}}
$$

Clearly, given the values of $T_{k}$, for $k \geq 2$, the other variables, which depend on $T_{k}$, are easily obtained from their definitions:

$$
\begin{aligned}
C_{k+1} & =c_{1} T_{k} \\
I_{k+2} & =c_{1} b\left(T_{k+1}-T_{k}\right)
\end{aligned}
$$

that is: 


$$
\begin{aligned}
C_{k+1} & =c_{1} \beta_{1} s_{1}^{k}+c_{1} \beta_{2} s_{2}^{k}+c_{1} T^{*} \\
I_{k+2} & =c_{1} b \beta_{1}\left(s_{1}^{k+1}-s_{1}^{k}\right)+c_{1} \beta_{2}\left(s_{2}^{k+1}-s_{2}^{k}\right)
\end{aligned}
$$

from the initial conditions $T_{0}$ and $T_{1}$ for $k=0$ and $k=1$, respectively, we have

$$
\begin{aligned}
C_{1} & =c_{1} T_{0} \\
I_{2} & =c_{1} b\left(T_{1}-T_{0}\right)
\end{aligned}
$$

and

$$
\begin{aligned}
C_{2} & =c_{1} T_{1} \\
I_{3} & =c_{1} b\left(T_{2}-T_{1}\right)
\end{aligned}
$$

When $\Delta=0$ (i.e. for $0<c_{1}<1$ and $b=b_{-}\left(c_{1}\right)$ ) there are two coincident real roots $s_{1}=s_{2}=s$ where $s=\frac{c_{1}(1+b)}{2}$ and then the general solution $T_{k}$, for $k \geq 2$, becomes as follows:

$$
T_{k}=\left(\beta_{1}+\beta_{2} k\right) s^{k}+T^{*}
$$

where

$$
\beta_{1}=\left(T_{0}-T^{*}\right), \beta_{2}=\frac{\left(T_{1}-T^{*}\right)-s\left(T_{0}-T^{*}\right)}{s}, s=\frac{c_{1}(1+b)}{2} .
$$

Differently, when the eigenvalues $s_{1}$ and $s_{2}$ are complex conjugate $(\Delta<0)$, that is for $0<c_{1}<1$ and $b_{-}\left(c_{1}\right)<b<\frac{1}{c_{1}}$ (yellow region in Fig. 1), with:

$$
s_{1}=\frac{c_{1}(1+b)+i \sqrt{4 c_{1} b-c_{1}^{2}(1+b)^{2}}}{2}, s_{2}=\frac{c_{1}(1+b)-i \sqrt{4 c_{1} b-c_{1}^{2}(1+b)^{2}}}{2}=\overline{s_{1}}
$$

having modulus $\sqrt{c_{1} b}$ so that

$$
s_{1}=\sqrt{c_{1} b}(\cos \alpha+i \sin \alpha), s_{2}=\sqrt{c_{1} b}(\cos \alpha-i \sin \alpha), \cos \alpha=\frac{c_{1}(1+b)}{2 \sqrt{c_{1} b}}
$$

then the general solution $T_{k}$, for $k \geq 2$, which is spiraling around the fixed point, becomes as follows:

$$
T_{k}=\left(\sqrt{c_{1} b}\right)^{k}\left(\beta_{1} \cos (k \alpha)+\beta_{2} \sin (k \alpha)\right)+T^{*}
$$

where

$$
\beta_{1}=\left(T_{0}-T^{*}\right), \beta_{2}=-\frac{\cos \alpha}{\sin \alpha}\left(T_{0}-T^{*}\right)+\frac{\left(T_{1}-T^{*}\right)}{\sqrt{c_{1} b} \sin \alpha}
$$

The other variables $C_{k}$ and $I_{k}$, which depend on $T_{k}$, are easily obtained from their definitions given in (12).

This original system by Samuelson has been considered also recently in Ortega and Barros (2020), where the authors claim to give the explicit solutions for $T_{k}$ (and also $C_{k}$ and $I_{k}$ ). However, their solution is not correct, since it does not correspond to the 
one given above, and there is no discussion with respect to the eigenvalues, complex conjugate or real (distinct or coincident).

We have so proved the following

Proposition 1 The Samuelson's model defined in (1), (2) and (3) with constant governmental expenditure $G_{k}=\bar{G}$ is economically meaningful for $0<c_{1}<1$ and $b>0$. The fixed point $T^{*}=\frac{\bar{G}}{1-c_{1}}$ is attracting for $0<c_{1}<1$ and $0<b<\frac{1}{c_{1}}$. In particular, it is an attracting node for $0<b<\frac{2-c_{1}-2 \sqrt{1-c_{1}}}{c_{1}}<1$, an attracting focus for $\frac{2-c_{1}-2 \sqrt{1-c_{1}}}{c_{1}}<b<\frac{1}{c_{1}}$. The analytic solution of the equation given in (4) as a function of two arbitrary initial conditions $T_{0}$ and $T_{1}$ for $k=0$ and $k=1$, respectively, are given in (10), (11) when the eigenvalues (8) are real and distinct, in (16) and (17) when the eigenvalues are real and coincident, in (20) and (21) when the eigenvalues are complex conjugate. The other variables $C_{k}$ and $I_{k}$ are obtained from their definitions given in (12).

\section{Results and discussion}

\subsection{Delayed Samuelson's Model and stability analysis}

The reformulated delayed version of Samuelson's model presented in Barros and Ortega (2019) is based on the following assumptions:

Assumption (1) is equivalent to Assumption (A). National income $T_{k}$ at time $k$ equals to the sum of three elements: consumption $C_{k}$, private investment $I_{k}$ and governmental expenditures assumed constant $\bar{G}$ (as in Assumption (D)):

$$
T_{k}=C_{k}+I_{k}+\bar{G}
$$

Assumption (2). Consumption $C_{k}$ at time $k$ is a linear function of the incomes of the two preceding periods:

$$
C_{k}=c_{1} T_{k-1}+c_{2} T_{k-2}
$$

where $c_{1}$ and $c_{2}$ are positive constant, $c_{1}>0, c_{2}>0$, and $0<c_{1}+c_{2}<1$. The governmental expenditures $\bar{G}$ in the model by Barros and Ortega (2019) are included in the consumption $C_{k}$, but since these are assumed constant, it does not differ from assuming them in the income $T_{k}$.

Assumption (3) is equivalent to Assumption (C). Private investment $I_{k}$ at time $k$, depends on consumption changes and on the positive accelerator factor $b>0$ :

$$
I_{k}=b\left(C_{k}-C_{k-1}\right)
$$

so that we get the following equation:

$$
I_{k}=b c_{1} T_{k-1}+b\left(c_{2}-c_{1}\right) T_{k-2}-b c_{2} T_{k-3}
$$

Summarizing, by using (22), (23) and (25) the national income is determined via the following third-order linear difference equation:

$$
T_{k}-c_{1}(1+b) T_{k-1}-\left[c_{2}+b\left(c_{2}-c_{1}\right)\right] T_{k-2}+b c_{2} T_{k-3}=\bar{G}
$$

or, equivalently, 


$$
T_{k+3}-c_{1}(1+b) T_{k+2}-\left[c_{2}+b\left(c_{2}-c_{1}\right)\right] T_{k+1}+b c_{2} T_{k}=\bar{G}
$$

Setting $T_{k}=T^{*}$ constant, from (26) we have the fixed point, given by

$$
T^{*}=\frac{\bar{G}}{1-\left(c_{1}+c_{2}\right)}
$$

and under the assumption on the parameters (being $0<c_{1}+c_{2}<1$ ) it is always positive and unique.

Notice that for $c_{1}+c_{2}=1$ the fixed point becomes infinite and for $c_{1}+c_{2}>1$ it is $T^{*}<0$, so that the condition $c_{1}+c_{2} \geq 1$ can be considered unfeasible, and we assume the constraint $0<c_{1}+c_{2}<1$, which, as we shall see, corresponds to the first condition for the stability region (associated with an eigenvalue equal to +1 ).

So it is now interesting to investigate the stability of the fixed point, as a function of the parameters (all positive), that is, under the given constraints:

$$
b>0,0<c_{1}+c_{2}<1
$$

Let us introduce the three-dimensional map, by defining $x_{k}=T_{k}$, $y_{k}=T_{k+1}=x_{k+1}, z_{k}=T_{k+2}=y_{k+1}$, so that we have the 3D linear system

$$
\left\{\begin{array}{c}
x_{k+1}=y_{k} \\
y_{k+1}=z_{k} \\
z_{k+1}=-b c_{2} x_{k}+\left[c_{2}+b\left(c_{2}-c_{1}\right)\right] y_{k}+c_{1}(1+b) z_{k}+\bar{G}
\end{array}\right.
$$

and in the 3D space $X=(x, y, z)$ the fixed point is

$$
X^{*}=\left(T^{*}, T^{*}, T^{*}\right)
$$

The coefficient matrix is given by

$$
A=\left[\begin{array}{ccc}
0 & 1 & 0 \\
0 & 0 & 1 \\
-b c_{2} & {\left[c_{2}+b\left(c_{2}-c_{1}\right)\right]} & c_{1}(1+b)
\end{array}\right]
$$

so that we recover the previous result, that is, from $\operatorname{det}(I-A)=1-\left(c_{1}+c_{2}\right)$ we have the condition on the invertibility of $(I-A)$, given by $c_{1}+c_{2} \neq 1$. The constant $\bar{G}$ is just a scaling factor, so it could be assumed $\bar{G}=1$, and, as in the original Samuelson's model, it only influences the value of the fixed point $T^{*}$.

The stability of the fixed point depends on the eigenvalues of the matrix $A$, that is, $X^{*}$ is attracting if all the eigenvalues (say $\left(\xi_{1}, \xi_{2}, \xi_{3}\right)$ ) of $A$ are smaller than 1 in absolute value. The eigenvalues are given by the roots of the characteristic polynomial:

$$
\mathcal{P}(\lambda)=\lambda^{3}+a_{1} \lambda^{2}+a_{2} \lambda+a_{3}=0
$$

where

$$
\begin{aligned}
& a_{1}=-c_{1}(1+b)=-\left(\xi_{1}+\xi_{2}+\xi_{3}\right) \\
& a_{2}=b c_{1}-c_{2}(1+b)=+\left(\xi_{1} \xi_{2}+\xi_{1} \xi_{3}+\xi_{2} \xi_{3}\right) \\
& a_{3}=b c_{2}=-\xi_{1} \xi_{2} \xi_{3}
\end{aligned}
$$


In a recent work (Gardini et al. 2021), it is shown that assuming that $\mathcal{P}(0)=a_{3}$ satisfies $\left|a_{3}\right|<1$, the stability conditions are as follows:

$$
\begin{aligned}
& \text { (i) }: \mathcal{P}(1)=1+a_{1}+a_{2}+a_{3}>0 \\
& \text { (ii) }: \mathcal{P}(-1)=1-a_{1}+a_{2}-a_{3}>0 \\
& \text { (iii) }: 1-a_{2}-a_{3}^{2}+a_{1} a_{3}>0
\end{aligned}
$$

In our case, the determinant is $\operatorname{Det}(A)=\mathcal{P}(0)=a_{3}=b c_{2}>0$ so that it is always positive, and the condition reduces to $b c_{2}<1$. So we have to consider the two conditions $0<c_{2}<\frac{1}{b}$ and $0<c_{2}<1-c_{1}$, that is:

$$
0<c_{2}<\min \left\{\frac{1}{b}, 1-c_{1}\right\}
$$

Substituting the expressions in (33) in conditions (34) we obtain:

$$
\begin{aligned}
& \text { (i) } 1-c_{1}-c_{2}>0 \\
& \text { (ii) } 1+(1+2 b)\left(c_{1}-c_{2}\right)>0 \\
& \text { (iii) } b^{2} c_{2}^{2}-c_{2}\left[(1+b)\left(1-b c_{1}\right)\right]-\left(1-b c_{1}\right)<0
\end{aligned}
$$

The equalities in the three conditions given in (36), coupled with (35), give the boundary of the stability region in the parameter space. Condition (i) is associated with an eigenvalue equal to 1 , condition (ii) is associated with an eigenvalue equal to -1 , and condition (iii) with complex conjugate eigenvalues in modulus equal to 1 . It follows that for any fixed value of the parameter $b, b>0$, we can have the boundaries of the stability region determined (besides $c_{i}>0$ ) by the conditions in (35) and

$$
\begin{aligned}
\text { (i) } c_{2} & <1-c_{1} \\
\text { (ii) } c_{2} & <c_{1}+\frac{1}{1+2 b} \\
\text { (iii) } c_{2} & <\widetilde{c_{2}}\left(b, c_{1}\right)
\end{aligned}
$$

where

$$
\widetilde{c_{2}}\left(b, c_{1}\right)=\frac{(1+b)\left(1-b c_{1}\right)+\sqrt{(1+b)^{2}\left(1-b c_{1}\right)^{2}+4 b^{2}\left(1-b c_{1}\right)}}{2 b^{2}}
$$

The boundaries of the region determined by the three conditions (i-iii) are given by curves in the parameter plane $\left(c_{1}, c_{2}\right)$ of equation:

$$
\begin{aligned}
& \left(r_{1}\right) c_{2}=1-c_{1} \\
& \left(r_{2}\right) c_{2}=c_{1}+\frac{1}{1+2 b} \\
& \left(r_{3}\right) c_{2}=\widetilde{c_{2}}\left(b, c_{1}\right)
\end{aligned}
$$

Let us first prove the following

Proposition 2 For any $b>0$ it holds that: 
the three curves of equation $c_{2}=\frac{1}{b}$ (i.e. $\left.\operatorname{Det}(A)=a_{3}=1\right),\left(r_{2}\right)$ and $\left(r_{3}\right)$ always intersect in the point $\left(c_{1}, c_{2}\right)=\left(\frac{1+b}{b(1+2 b)}, \frac{1}{b}\right)=: R$;

the curve $\left(r_{3}\right)$ always intersects the $c_{1}$-axis in the point $\left(c_{1}, 0\right)=\left(\frac{1}{b}, 0\right)$;

the two straight lines $\left(r_{1}\right)$ and $\left(r_{2}\right)$ always intersect in the point $\left(c_{1}, c_{2}\right)=\left(\frac{b}{1+2 b}, \frac{1+b}{1+2 b}\right)=: Q$;

at $\quad b=b^{*}, \quad b^{*}=\frac{1+\sqrt{5}}{2} \simeq 1.618033989, \quad$ it $\quad$ is $\quad R=Q ; \quad$ for $\quad b<b^{*} \quad$ it is $c_{2}(R)=\frac{1}{b}>c_{2}(Q)=\frac{1+b}{1+2 b}$; for $b>b^{*}$ it is $c_{2}(R)=\frac{1}{b}<c_{2}(Q)=\frac{1+b}{1+2 b}$.

Proof The straight lines $c_{2}=\frac{1}{b}$ and $\left(r_{2}\right)$ are intersecting in $\left(c_{1}, c_{2}\right)=\left(\frac{1+b}{b(1+2 b)}, \frac{1}{b}\right)$, Setting $c_{2}=\frac{1}{b}$ in condition (iii) in (36) it comes immediately that $\left(r_{3}\right)$ holds iff $c_{1}=\frac{1+b}{b(1+2 b)}$. The intersection point of the two straight lines $\left(r_{1}\right)$ and $\left(r_{2}\right)$ also comes soon by direct computation. Finally by direct computation we have $\frac{1}{b} \gtreqless \frac{1+b}{1+2 b}$ for $b \lesseqgtr b^{*}$.

To comment the stability region of the fixed point, let us distinguish the two cases $0<b \leq 1$ and $b>1$, which have different properties in the parameter plane $\left(c_{1}, c_{2}\right)$.

(I) $0<b \leq 1$

For $b \leq 1$ the triangle bounded by the line $c_{2}=1-c_{1}$ (intersecting the axes in the points $(0,1)$ and $(1,0))$ is below the line $c_{2}=\frac{1}{b} \geq 1$, having a contact point in $(0,1)$ only for $b=1$.

The segment of $\left(r_{1}\right)$ connecting the two points $(1,0)$ and $(0,1)$ gives the boundary of the region satisfying $0<c_{1}+c_{2}<1$, in which condition (i) is satisfied.

The segment of $\left(r_{2}\right)$ connecting the two points $\left(\frac{-1}{1+2 b}, 0\right)$ and $\left(0, \frac{1}{1+2 b}\right)$ is inside the triangle bounded by the segment of $c_{2}=1-c_{1}$ so that it is an active constraint. Notice that the point $\left(0, \frac{1}{1+2 b}\right)$ approaches $(0,1)$ as $b$ tents to zero. (That is, increasing $b$ from zero the stability region decreases, or, equivalently, decreasing $b$ from 1 the stability region increases).

From the properties given in Proposition 2, the curve in the first quadrant of the parameter plane $\left(c_{1}, c_{2}\right)$ of equation $c_{2}=\widetilde{c_{2}}\left(c_{1}\right)$ is always external to the stability region, which is only bounded by segments of the straight lines $\left(r_{1}\right)$ and $\left(r_{2}\right)$. Some examples are shown in Fig. 2.

It follows that changing the parameters inside the stability region, either it is crossed the boundary at which the fixed point becomes infinite (segment of $\left(r_{1}\right)$ ), or it is crossed the boundary at which one eigenvalue is equal to -1 (segment of $\left(r_{2}\right)$ ).

(II) $b>1$

Increasing $b$ from 1 , the curve of equation $c_{2}=\widetilde{c_{2}}$ moves in the opposite direction (since now it is $\left.\frac{1}{b}<1\right)$, intersecting the segment of $\left(r_{1}\right)$ on the boundary of the stability region as long as it holds $1<b<b^{*}$, examples are shown in Fig. 3a, b, while for $b>b^{*}$ the straight line $\left(r_{1}\right)$ is external to the stability region, whose boundary is given by a segment of $\left(r_{2}\right)$ and a segment of $\left(r_{3}\right)$, an example is shown in Fig. 3c.

For the characteristic polynomial in (32) it is known (see e.g. Gardini et al. (2021)) that considering the discriminant

$$
D=4 a_{2}^{3}-a_{1}^{2} a_{2}^{2}+4 a_{1}^{3} a_{3}-18 a_{1} a_{2} a_{3}+27 a_{3}^{2}
$$

then for $D<0$ three real roots exist, at $D=0$ two real roots merge and for $D>0$ there are two complex conjugate roots and a real one. By using the expressions given in (33) 

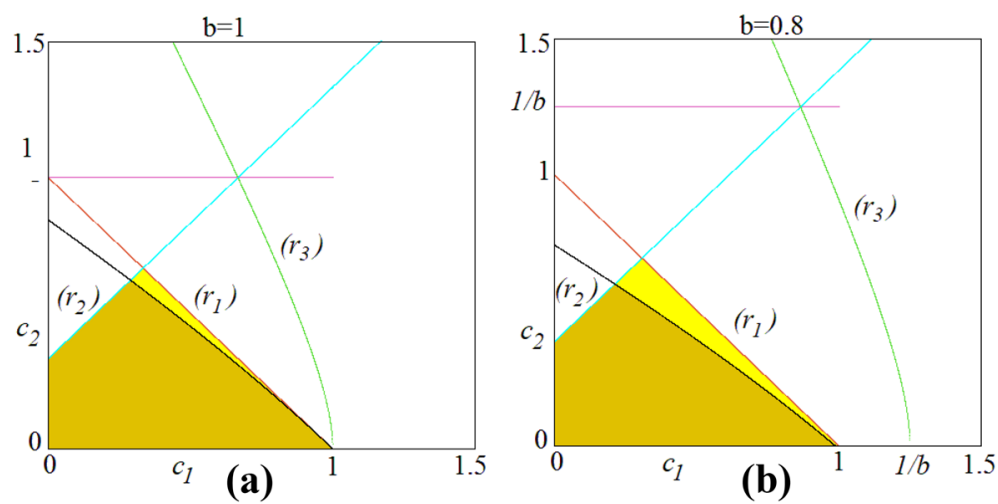

Fig. 2 In color the stability region of the fixed point $T^{*}$ for $0<b \leq 1$ in the parameter plane $\left(c_{1}, c_{2}\right)$. In a at $b=1$. In $\mathbf{b}$ at $b=0.8$. The black curve corresponds to the discriminant $D=0$ given in (40). In the orange region a pair of complex eigenvalues exists. In the yellow region the three eigenvalues are real

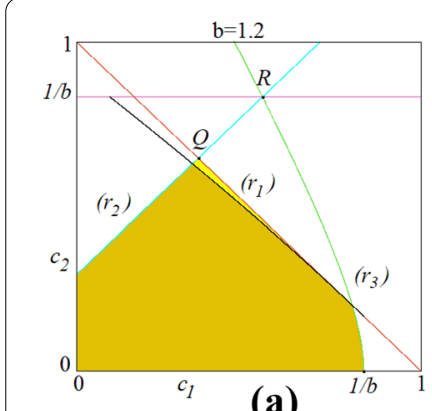

(a)

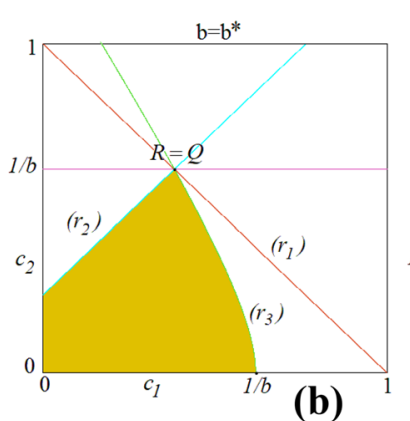

(b)

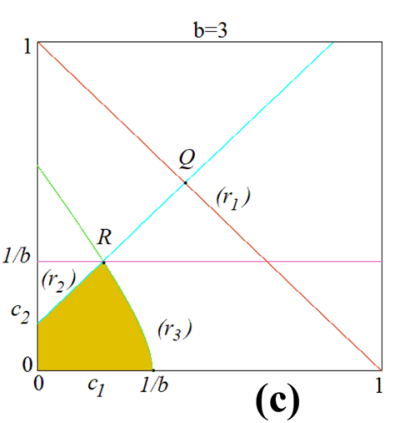

(c)

Fig. 3 In color the stability region of the fixed point $T^{*}$ for $b>1$ is shown in the parameter plane $\left(c_{1}, c_{2}\right)$. In $\mathbf{a}$ at $b=1.2$, the black curve denotes the discriminant $D=0$ given in (40). $\ln \mathbf{b}$ at $b=b^{*}$. In $\mathbf{c}$ at $b=3$. In the orange region a pair of complex eigenvalues exist. In the yellow region the three eigenvalues are real

we have numerically evaluated the discriminant $D$ and shown the graph of $D=0$ in Figs. 2, 3. As we can see, the stability region is mainly associated with a pair of complex eigenvalues, and for $b>b^{*}$ it is always so.

Notice that for a specific set of parameters we can numerically evaluate the eigenvalues and then, since the model is linear, we can easily write the explicit solution analytically as a functions of three initial conditions.

As an example, considering the values $b=1.5, c_{1}=0.62, c_{2}=0.1$ which is close to the boundary of $\left(r_{3}\right)$, the fixed point $T^{*}=\frac{\bar{G}}{1-\left(c_{1}+c_{2}\right)}=3.571429 \bar{G}$ is attracting, with one real negative eigenvalue and two complex conjugate ones, so that we have oscillations converging to the equilibrium, as shown in Fig. 4.

As a final remark, we can state that the delayed version of Samuelson's model reduces the stability region of the fixed point.

\subsection{Nonlinear Samuelson's model}

Let us come back to the original Samuelson's model, where the governmental expenditure may be not constant, $G_{k}$, and combine this with the delayed version of Samuelson's 


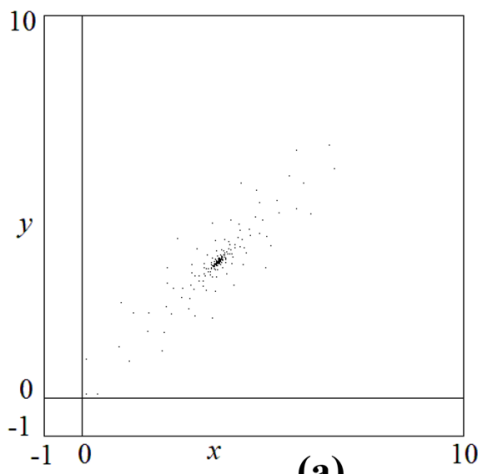

(a)

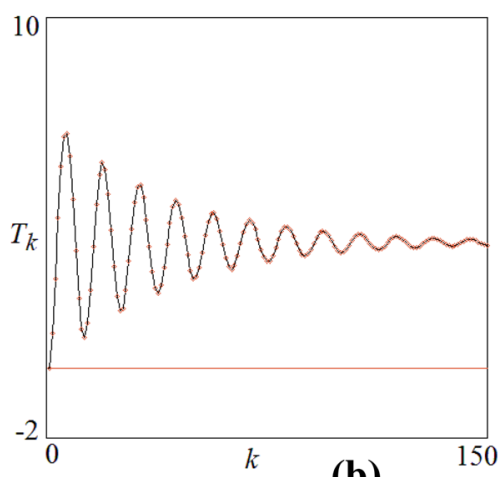

(b)

Fig. 4 In a trajectory of the third-order difference equation in the plane $(x, y)=\left(T_{k}, T_{k+1}\right)$ for $b=1.5$, $c_{1}=0.62, c_{2}=0.1, \bar{G}=1$. In $\mathbf{b}$ the versus time trajectory of $T_{k}$ for $2<k<150$

model by Barros and Ortega (2019), by using $G_{k}$ in (22) in place of a constant value, keeping all the other assumptions as in the delayed model of the previous section.

The governmental expenditure $G_{k}$ may consist of a constant amount $\bar{G}$ (which includes, for example, fixed essential costs and other fixed expenses), and a part which depends on the income of the previous period, taking values in a bounded range, for example given by $\gamma \arctan \left(T_{k-1}\right)$, where $\gamma$ is a parameter governing the width of the range, thus leading to our Assumption (4):

$$
G_{k}=\bar{G}+\gamma \arctan \left(T_{k-1}\right)
$$

This assumption is consistent, for instance, with the well known Wagner's hypothesis (Wagner 1890). According to this there is a causation that runs from economic growth to growth in government expenditure. This hypothesis has been tested empirically in several countries (see Gemmell 1990; Ansari 1993; Courakis et al. 1993; Dollery and Singh 1998; Peacock and Scott 2000, among the others). Thus, we get the following nonlinear equation

$$
T_{k+3}-c_{1}(1+b) T_{k+2}-\left[c_{2}+b\left(c_{2}-c_{1}\right)\right] T_{k+1}+b c_{2} T_{k}=\bar{G}+\gamma \arctan \left(T_{k+2}\right)
$$

leading to the three-dimensional nonlinear system

$$
\left\{\begin{array}{c}
x_{k+1}=y_{k} \\
y_{k+1}=z_{k} \\
z_{k+1}=-b c_{2} x_{k}+\left[c_{2}+b\left(c_{2}-c_{1}\right)\right] y_{k}+c_{1}(1+b) z_{k}+\gamma \arctan \left(z_{k}\right)+\bar{G}
\end{array}\right.
$$

The fixed point of the nonlinear system is now given by the solution of the following nonlinear equation:

$$
T^{*}\left(1-c_{1}-c_{2}\right)-\bar{G}=\gamma \arctan \left(T^{*}\right)
$$

and it is easy to see that it can be considered as the intersection point of an increasing function $\left(\gamma \arctan \left(T^{*}\right)\right)$ with a straight line $\left(T^{*}\left(1-c_{1}-c_{2}\right)-\bar{G}\right)$ with positive slope and negative offset, as qualitatively shown in Fig. 5 (where the function $\gamma \arctan \left(T^{*}\right)$ 


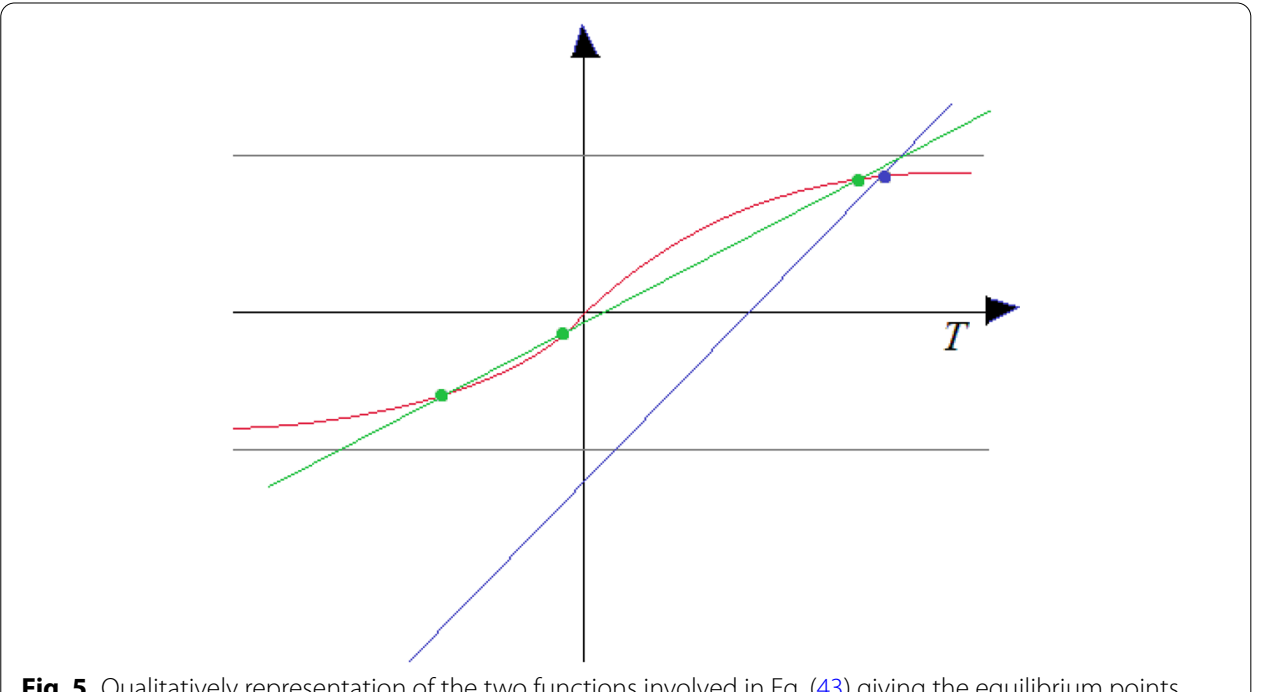

Fig. 5 Qualitatively representation of the two functions involved in Eq. (43) giving the equilibrium points

is shown in red, while in green and in blue are shown two versions of the function $\left(T^{*}\left(1-c_{1}-c_{2}\right)-\bar{G}\right)$.

Thus, we may expect a unique fixed point in the positive side, as shown by the blue circle in Fig. 5, which is an intersection of the red curve with the blue straight line, but it is also possible to have three fixed points, as shown by the green circles in Fig. 5 which are the intersections of the red curve with the green straight line. However, this second situation may be considered unfeasible, since the two more fixed points must belong to the negative side.

Let us consider the positive fixed point. Since we cannot have its value analytically, the local stability analysis cannot be performed analytically. However, by using numerical simulations, we can see that the stability region is quite similar to the one of the linear case, as shown in Fig. 6a. ${ }^{1}$ In that figure, at a value of $b$ such that $0<b<1$, we can see that the upper left boundary corresponds to a flip bifurcation (as in the linear case of the previous section). An example soon after the flip bifurcation is shown in Fig. $6 \mathrm{~b}$ projected in the $(x, y)$, where the unstable set of the fixed point converges to an attracting 2-cycle. In the upper right boundary the bifurcation is related to to the fixed point approaching infinity, similarly to the linear case of the delayed model considered in the previous section.

Also when $b>1$ we have a stability region quite similar to the one observed in the linear case, as shown in Fig. 7a, with the upper left boundary leading to a flip bifurcation with a behavior similar to the one observed above (as shown in Fig. 7b), and the right side of the boundary of the stability region now includes also a border associated with a Neimark-Sacker bifurcation.

\footnotetext{
${ }^{1}$ Figures 6a and 7a are obtained numerically considering a grid in the parameter plane, and for each point of the parameter plane we take an initial condition close to the fixed point, numerically obtained, and we check the behavior of the trajectory, which may be divergent, convergent to the fixed point or a 2-cycle or to something else (as a closed invariant curve).
} 


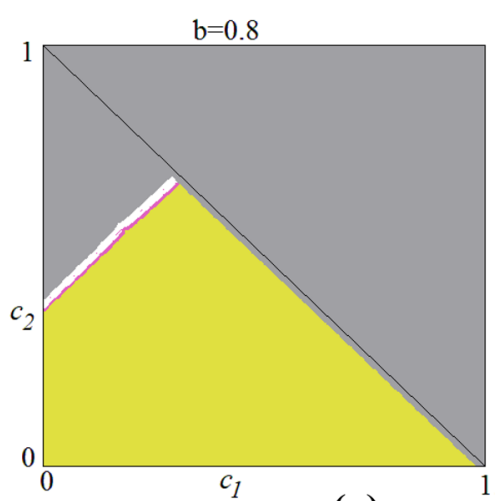

(a)

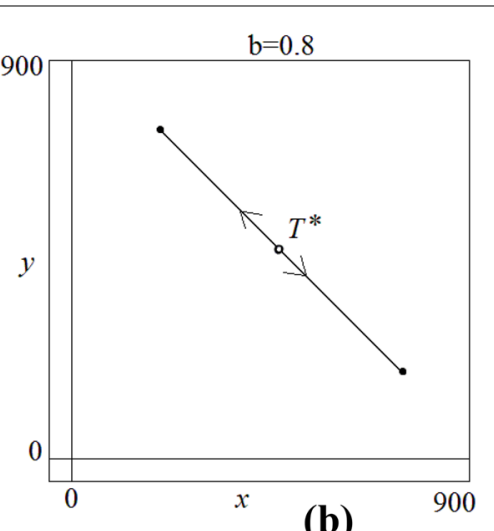

(b)

Fig. 6 In a the yellow region represents the stability region of the fixed point $T^{*}$ in the parameter plane $\left(c_{1}, c_{2}\right)$ at $b=0.8, \bar{G}=100, \gamma=1$, initial condition $(400,400,400)$. In $\mathbf{b}$ projection of the trajectory of the nonlinear model in the plane $(x, y)=\left(T_{k}, T_{k+1}\right)$ showing the attracting 2 -cycle existing at $b=0.8, c_{1}=0.2$, $c_{2}=0.584618$

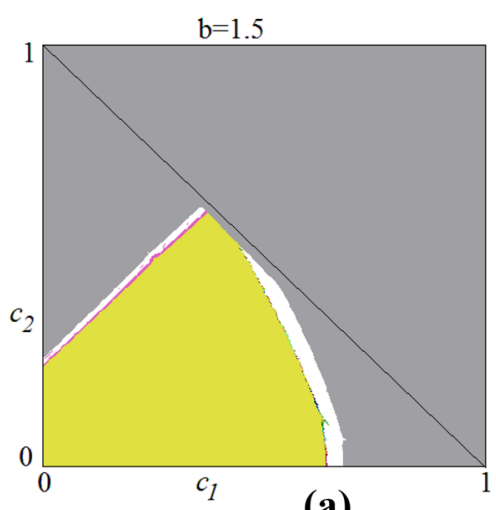

(a)

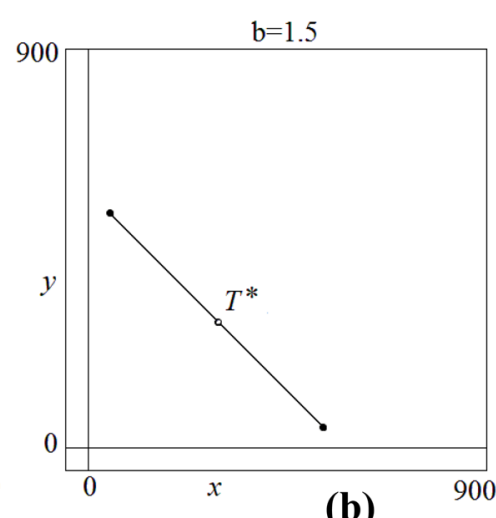

(b)

Fig. 7 In a the yellow region represents the stability region of the fixed point $T^{*}$ in the parameter plane $\left(c_{1}, c_{2}\right)$ at $b=1.5, \bar{G}=100, \gamma=1$, initial condition $(400,400,400)$. In $\mathbf{b}$ projection of the trajectory of the nonlinear model in the plane $(x, y)=\left(T_{k}, T_{k+1}\right)$ showing the attracting 2 -cycle existing at $b=1.5, c_{1}=0.2$, $c_{2}=0.45001$

It is worth noting that in the nonlinear model global bifurcations may occur, so that, also in the case of a unique fixed point, other attracting sets may appear. An example is shown in Fig. 8a, and enlarged in Fig. 8b where the attracting fixed point is close to the Neimark-Sacker bifurcation, but it coexists with another attractor, belonging to a closed invariant curve. From the enlargement we can argue that it is a cycle of very high period. The global effects involve regions in the phase space far from the equilibrium of interest, and it is relevant in order to determine the width of the basin of attraction of the equilibrium. The section of the basin of attraction on the plane $z=T^{*}$ is shown in Fig. 8c, and in the same figure we also have reported the projection of the two attractors. In it, yellow points converge to the fixed point while red points converge to the wide cycle, showing that the separator is probably a repelling closed invariant curve. As in fact, approaching the Neimark-Sacker bifurcation value, this curve shrinks to the fixed point in a subcritical bifurcation. 


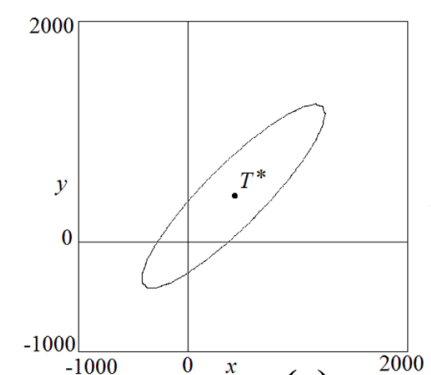

(a)

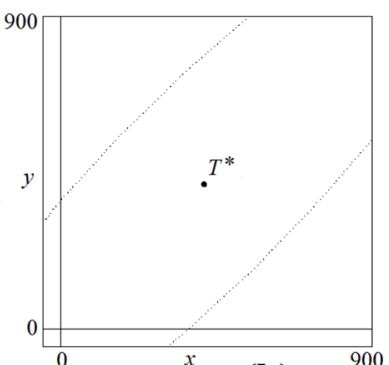

(b)

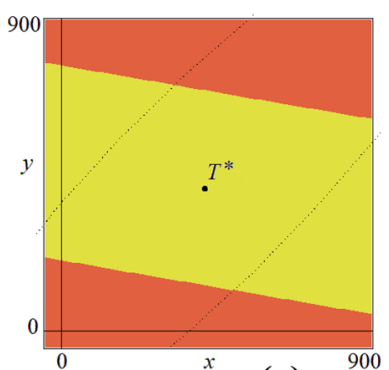

(c)

Fig. 8 In a projection of the trajectory of the nonlinear model in the plane $(x, y)=\left(T_{k}, T_{k+1}\right)$ for $b=1.5$, $c_{1}=0.6545, c_{2}=0.1, \bar{G}=100, \gamma=1$, initial condition $(400,400,400)$. In $\mathbf{b}$ its enlargement. In $\boldsymbol{c}$ the basin of attraction on the plane $z=T^{*}$

\section{Conclusions}

In the present work we have shown how strong is the ability of the Samuelson's model to represent stable oscillations converging to an equilibrium value. This has been shown in three cases. Namely, in Sect. 1 we have reconsidered the classical Samuelson's multiplier-accelerator model for national economy with constant governmental expenditure. The stability region has been analytically determined as well as its analytic solution in all the possible cases of real or complex eigenvalues. In Sect. 2 we have reconsidered the delayed version of Samuelson's model presented in Barros and Ortega (2019), performing the analysis of the stability region as a function of the three parameters, showing that indeed the stability region is mainly related to oscillatory behavior. In Sect. 3 we have proposed a simple nonlinear reformulation of the original Samuelson's model, with non constant governmental expenditure, maintaining the delayed version presented in Barros and Ortega (2019). Here we have performed a numerical investigation of the stability region, showing that it is quite similar to the one presented in Sect. 2, and also that the dynamics are mainly oscillating towards the stable equilibrium.

Our analysis shows that the seminal work of Samuelson (1939), and simple modifications of it, may give powerful tools in the study of the business cycles. A calibration of the parameters with realistic outcomes is left for future work.

\section{Acknowledgements}

The authors conducted this study within the research project on Models of Behavioral Economics for Sustainable Development, at the Department of Economics, Society, Politics (DESP) of the University of Urbino. metto quello del paper

Authors' contributions

Both authors contributed equally to the writing of this paper. All authors read and approved the final manuscript.

\section{Funding}

Not applicable.

Availability of data and materials

Not applicable.

\section{Declarations}

Ethics approval and consent to participate

Both authors approved the participation

Consent for publication

Both authors read and approved the final manuscript 


\section{Competing interests}

The authors declare that there is no conflict of interest

\section{Author details}

${ }^{1}$ Department DiMSEFA Università Cattolica del Sacro Cuore, Via Necchi, n.9, 20123 Milano, Italy. ${ }^{2}$ Department DESP,

University of Urbino, Urbino, Italy.

Received: 27 June 2020 Revised: 9 June 2021 Accepted: 10 June 2021

Published online: 28 June 2021

\section{References}

Ansari MI (1993) Testing the relationship between government expenditure and national income in Canada, employing Granger causality and cointegration analysis. Manag Finance 19(7):31-46

Barros MF, Ortega F (2019) An optimal equilibrium for a reformulated Samuelson economic discrete time system. J Econ Struct 8:29. https://doi.org/10.1186/s40008-019-0162-2

Bischi Gl, Matsumoto A, Sanchez-Carrera E (2019) Special issue on "Nonlinear Social Dynamics". Structural change and economic dynamics

Courakis AS, Moura-Roque F, Tridimas G (1993) Public expenditure growth in Greece and Portugal: Wagner's law and beyond. Appl Econ 25(1):125-134

Dalla E, Varelas E (2016) An economic model for the interpretation of business cycles and the efficiency of monetary policy. J Econ Asymmetries 14:29-38

Dassios I (2018) A practical formula of solutions for a family of linear non-autonomous fractional nabla difference equations. J Comput Appl Math 339:317-328

Dassios I, Baleanu D (2018) Caputo and related fractional derivatives in singular systems. Appl Math Comput 337:591-606

Dassios I, Devine M (2016) A macroeconomic mathematical model for the national income of a union of countries with interaction and trade. J Econ Struct 5:18

Dassios I, Zimbidis A (2014) The classical Samuelson's model in a multi-country context under a delayed framework with interaction. Dyn Contin Discret Impuls Syst Ser B Appl Algorithms 21(4-5b):261-274

Dassios I, Zimbidis A, Kontzalis C (2014) The delay effect in a stochastic multiplier-accelerator model. J Econ Struct 3:7

Dollery B, Singh S (1998) A note on the empirical analysis of Wagner's law. Econ Anal Policy 28(2):245-257

Elaydi S (2005) An introduction to difference equations. Springer, New-York

Gardini L, Schmitt N, Sushko I, Tramontana F, Westerhoff F (2021) Necessary and sufficient conditions for the roots of a cubic polynomial and bifurcations of codimension-1, -2, -3 for 3D maps. J Differ Eq Appl 2021:1-22

Gemmell N (1990) Wagner's Law. Relative prices and the size of the Public Sector. Manchester Sch Econ Soc Stud 58(4):361-77

Gumowsky I, Mira C (1980) Recurrences and discrete dynamical systems. Springer, New-York

Hommes CH (1991) Chaotic dynamics in economic models. Wolters-Noodhoff, Groningen

Kostarakos I, Kotsios S (2017) Feedback policy rules for government spending: an algorithmic approach. J Econ Struct 6(1):1-10

Ortega F, Barros MF (2020) The Samuelson macroeconomic model as a singular linear matrix difference equation. J Econ Struct 9:36. https://doi.org/10.1186/s40008-020-00207-3

Peacock A, Scott A (2000) The curious attraction of Wagner's Law, Public Choice 102:1-17

Puu T $(1989,1997,2003)$ Nonlinear economic dynamics. Springer, Berlin

Puu T, Gardini L, Sushko I (2005) A Hicksian multiplier-accelerator model with floor determined by capital stock. J Econ Behav Organ 56:331-348

Rosser JB (2000) From catastrophe to chaos: a general theory of economic discontinuities. Academic Publishers, Boston Samuelson PA (1939) Interactions between the multiplier analysis and the principle of acceleration. Rev Econ Stat 21:75-78; reprinted in Readings in Business Cycle Theory, Blakiston, Philadelphia, 1944

Wagner A (1890) Finanzwissenschaft, 3a edition Leipzig. In: Musgrave RA, Peacock AT (eds) Classics in the theory of public finance. Macmillan, London

Westerhoff FH (2006) Samuelson's multiplier-accelerator model revisited. Appl Econ Lett 56:86-92

\section{Publisher's Note}

Springer Nature remains neutral with regard to jurisdictional claims in published maps and institutional affiliations. 